\title{
Autonomous multi-joint soft exosuit with augmentation-power-based control parameter tuning reduces energy cost of loaded walking
}

Sangjun Lee ${ }^{1,2}$, Jinsoo Kim ${ }^{1,2+}$, Lauren Baker ${ }^{1,2+}$, Andrew Long ${ }^{1,2}$, Nikos Karavas ${ }^{1,2}$, Nicolas Menard ${ }^{1,2}$, Ignacio Galiana ${ }^{1,2}$ and Conor J. Walsh ${ }^{1,2^{*}}$

\begin{abstract}
Background: Soft exosuits are a recent approach for assisting human locomotion, which apply assistive torques to the wearer through functional apparel. Over the past few years, there has been growing recognition of the importance of control individualization for such gait assistive devices to maximize benefit to the wearer. In this paper, we present an updated version of autonomous multi-joint soft exosuit, including an online parameter tuning method that customizes control parameters for each individual based on positive ankle augmentation power.

Methods: The soft exosuit is designed to assist with plantarflexion, hip flexion, and hip extension while walking. A mobile actuation system is mounted on a military rucksack, and forces generated by the actuation system are transmitted via Bowden cables to the exosuit. The controller performs an iterative force-based position control of the Bowden cables on a step-by-step basis, delivering multi-articular (plantarflexion and hip flexion) assistance during push-off and hip extension assistance in early stance. To individualize the multi-articular assistance, an online parameter tuning method was developed that customizes two control parameters to maximize the positive augmentation power delivered to the ankle. To investigate the metabolic efficacy of the exosuit with wearer-specific parameters, human subject testing was conducted involving walking on a treadmill at $1.50 \mathrm{~m} \mathrm{~s}^{-1}$ carrying a $6.8-\mathrm{kg}$ loaded rucksack. Seven participants underwent the tuning process, and the metabolic cost of loaded walking was measured with and without wearing the exosuit using the individualized control parameters.
\end{abstract}

Results: The online parameter tuning method was capable of customizing the control parameters, creating a positive ankle augmentation power map for each individual. The subject-specific control parameters and resultant assistance profile shapes varied across the study participants. The exosuit with the wearer-specific parameters significantly reduced the metabolic cost of load carriage by $14.88 \pm 1.09 \%\left(P=5 \times 10^{-5}\right)$ compared to walking without wearing the device and by $22.03 \pm 2.23 \%\left(P=2 \times 10^{-5}\right)$ compared to walking with the device unpowered.

Conclusion: The autonomous multi-joint soft exosuit with subject-specific control parameters tuned based on positive ankle augmentation power demonstrated the ability to improve human walking economy. Future studies will further investigate the effect of the augmentation-power-based control parameter tuning on wearer biomechanics and energetics.

Keywords: Exosuit, Assistance, Control, Tuning, Augmentation power, Metabolic cost

\footnotetext{
* Correspondence: walsh@seas.harvard.edu

† Jinsoo Kim and Lauren Baker contributed equally to this work.

${ }^{1}$ John A. Paulson School of Engineering and Applied Sciences, Harvard

University, Cambridge, MA, USA

${ }^{2}$ Wyss Institute for Biologically Inspired Engineering, Harvard University,

Cambridge, MA, USA
}

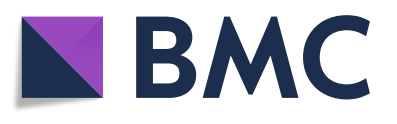

(c) The Author(s). 2018 Open Access This article is distributed under the terms of the Creative Commons Attribution 4.0 International License (http://creativecommons.org/licenses/by/4.0/), which permits unrestricted use, distribution, and reproduction in any medium, provided you give appropriate credit to the original author(s) and the source, provide a link to the Creative Commons license, and indicate if changes were made. The Creative Commons Public Domain Dedication waiver (http://creativecommons.org/publicdomain/zero/1.0/) applies to the data made available in this article, unless otherwise stated. 


\section{Introduction}

Lower-limb assistive devices have been designed to assist with human locomotion [1-12]. Recently, different groups have used rigid but lightweight mechanisms to create low-profile exoskeletons assisting with a specific target joint, and studies have shown that these devices may substantially reduce the energy cost of loaded [6] and unloaded [7-12] walking. For example, Lee et al. showed that their hip exoskeleton reduced the metabolic cost of walking by $21 \%$ compared to walking without wearing the device [12]. For ankle, Mooney et al. reported an 11\% net benefit for walking [10] and an $8 \%$ net benefit for load carriage [6] using their autonomous ankle exoskeleton.

Our group has been developing soft exosuits that use functional textiles to anchor to the body and deliver assistance in parallel with the underlying muscles [13-20]. In studies with tethered versions of the device, exosuits have been shown to significantly reduce the energy cost of regular walking [17, 20], walking with load [16, 19], and running [18]. For an autonomous version, Panizzolo et al. showed a $7 \%$ net metabolic reduction for loaded walking compared to equivalent-mass-removed condition (walking with the device unpowered but removing the equivalent mass of the device) [15].

Over the past few years, there has been a growing recognition on the importance of control individualization for such gait assistive devices to maximize one's benefit; however, only a few studies so far have investigated methods to systematically customize the controller of assistive devices. Conventionally, researchers have used manual tuning to individualize the assistance of exoskeletons [12] or powered prostheses [3], where the wearer or an external operator subjectively tunes the control parameters based on the user's perception or the observation of gait kinematics/kinetics. A challenge with a manual parameter tuning process is that it can involve a significant level of human subjective intervention, thus requiring expert knowledge and experience with the hardware. A more recent approach is human-in-the-loop optimization, where an optimization algorithm finds the optimal parameters that maximize one's metabolic benefit, estimating the wearer's instantaneous metabolic cost while walking [11, 20-22]. This approach holds advantages in that it automatically optimizes control parameters by directly monitoring the user's metabolic cost; however, the current approach requires a user to wear respiratory measurement equipment throughout the process. The field of prosthetics has made efforts to bridge the gap between these two approaches [23-27]. Researchers have derived dynamic models of locomotion with specific types of powered prostheses and used computational algorithms, such as supervised learning [24], extremum seeking controller (ESC) [25], or adaptive dynamic programming (ADP) [26], to find optimal impedance control parameters in the model for each individual.
Among them, Huang et al. suggested a method called cyber-expert tuning system for a powered knee prosthesis, where they implemented several decision rules of manual tuning into a computational algorithm based on data from the device's own wearable sensors [27]. The approach of performing automatic parameter tuning with only device sensors is appealing as it opens the door to this being performed outside of a lab setting. However, it remains unclear how this approach can be applied to the devices augmenting the gait of healthy individuals, because it is currently unclear what may be proxy objective metrics for metabolic cost and how those metrics can be measured by body-worn sensors. Therefore, if a control tuning method can be developed based on an objective function that is easily measurable and strongly correlated with metabolic cost, it may greatly improve the energetic efficacy of a gait assistive device for healthy individuals.

In this paper, we present an updated version of the autonomous multi-joint soft exosuit aimed at overground walking in outdoor settings [28]. In addition, we propose an online parameter tuning method that automatically customizes assistance based on the positive power delivered to the ankle by the exosuit. This is based on the assumption that a positive correlation exists between the positive ankle augmentation power and the corresponding metabolic benefit $[6,10,19,29-33]$. Given that this proxy objective metric can easily be measured by wearable sensors, we believe this augmentation-power-based parameter tuning approach holds a promise, given the desire to enable control individualization in unconstrained environments. Additionally, we present results from human subject testing demonstrating the metabolic efficacy of the soft exosuit with the subject-specific control parameters during loaded walking.

\section{Methods \\ Soft exosuit}

The multi-joint soft exosuit is designed to assist with plantarflexion, hip flexion, and hip extension while walking [28]. As shown in Fig. 1a, the exosuit apparel components consist of a waist belt, two thigh pieces, two calf wraps, two dynamic multi-articular straps connecting the front of the waist and the back of the calf, and two boot covers wrapping around the wearer's ankle. The multi-articular strap is designed to distribute assistive forces applied at the ankle between the calf wrap and the waist belt [28]. The total mass of all suit components for a size medium is $1.1 \mathrm{~kg}$, including two metal brackets bolted to the back of military boots. As shown in Fig. 1b, this textile architecture creates two different load paths on each leg: a multi-articular load path assisting with plantarflexion and hip flexion during push-off and a hip extension load path assisting with hip extension in early stance. 

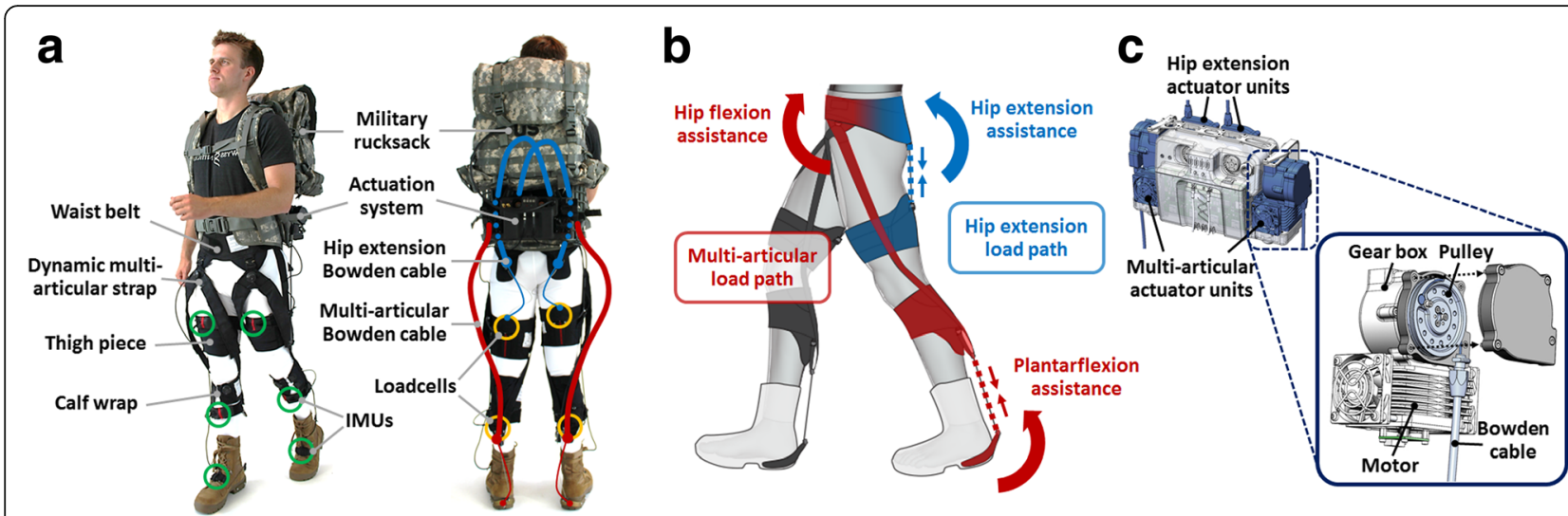

Fig. 1 The autonomous multi-joint soft exosuit used in this study. a The apparel components and the hardware implementation. Bowden cable routings for multi-articular assistance and hip extension assistance are indicated by red and blue lines, respectively (thick lines: cable sheaths, thin lines: inner cables). Sensor placements for IMUs and loadcells are marked by green and yellow circles, respectively. $\mathbf{b}$ Two load paths specified by the textile architecture: multi-articular load path assisting with plantarflexion and hip flexion (highlighted in red) and hip extension load path assisting with hip extension (highlighted in blue). c A 3-D CAD model of the mobile actuation system consisting of four independent actuator units (highlighted in blue) and an exploded view of a multi-articular actuator unit

\section{Hardware implementation}

A mobile actuation system was developed to generate assistive forces and mounted at the lower back of a military rucksack [28]. As shown in Fig. 1c, the actuation system consists of four independent actuator units, two for multi-articular load path and the other two for hip extension load path. Each actuator unit is comprised of an Emoteq frameless 6-pole motor (Allied Motion Inc., Amherst, NY, USA), a Spiroid helicon gear box (38:1 gearing ratio for multi-articular actuator units and 36:1 for hip extension actuator units; Illinois Tool Works, Inc., Glenview), and a 55-mm diameter multi-wrap pulley. The forces generated by the actuation system are transmitted to the exosuit via Bowden cables; when the motor retracts the Bowden cable, the distance between two attachment points on the exosuit is shortened, creating assistive forces along the corresponding load path. The actuation system including Bowden cables weighs $5.9 \mathrm{~kg}$, and a $48 \mathrm{~V}-8 \mathrm{~A} \cdot \mathrm{hr}$. Li-Po battery pack $(2.0 \mathrm{~kg})$ stowed in the rucksack was used to power the actuation system that would be sufficient for approximately $8 \mathrm{~km}$ of continuous walking operation.

On each leg, a linear daisy-chain harness including three inertial measurement units (IMU; MTi-3 AHRS; Xsens Technologies B.V., Enschede, Netherlands) and two load cells (LSB200; Futek Advanced Sensor Technology Inc., Irvine, CA, USA) was placed to collect real-time data from the exosuit and the wearer. As shown in Fig. 1a, the IMUs were attached to the wearer's thigh, shank, and foot to measure the sagittal-plane orientation and the angular velocity of each segment, while the load cells were mounted in series with the Bowden cables to monitor the level of assistive force delivered to the wearer through the exosuit. The full sensor harnesses including all sensors weigh $0.3 \mathrm{~kg}$.

\section{Biologically inspired control}

As with the previous-version exosuits [34, 35], the controllers for the multi-articular and hip extension load paths both performed a force-based position control of the Bowden cables to generate assistive forces. Inspired by biological behavior of the target joints, the controller applies the assistance by retracting the Bowden cable during a target period within a walking cycle. As shown in Figs. 2 and 3, the cable position

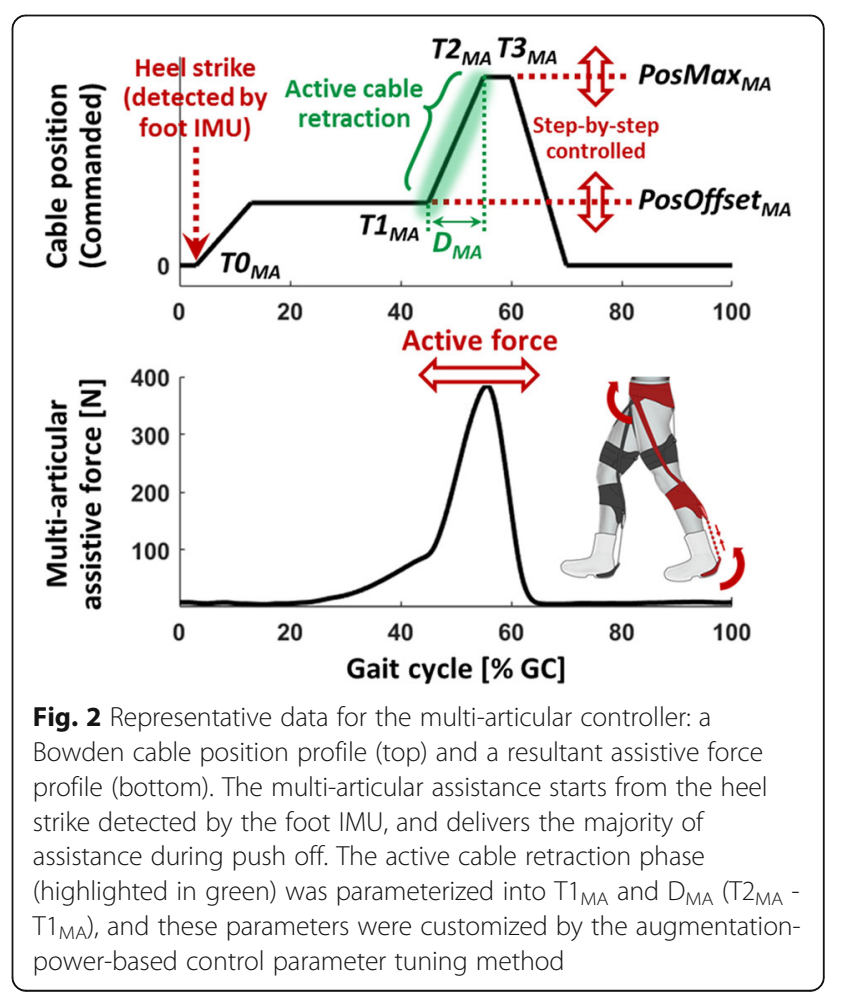




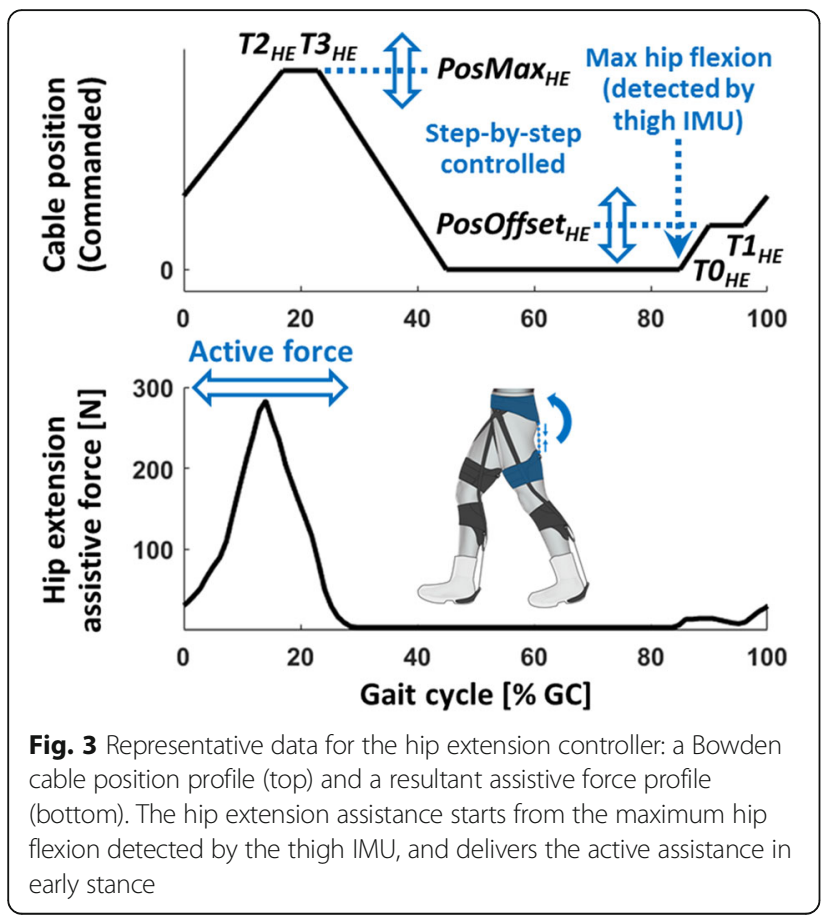

profiles were defined by four timing parameters, $\mathbf{T} 0, \boldsymbol{T} 1, \mathbf{T} 2$, and $T 3$, which are represented as percentage of a gait cycle (\% GC) and two cable position parameters, PosOffset and PosMax, which are iteratively adjusted on a step-by-step basis $[34,35]$ :

- T0: the start timing of the controller within a gait cycle.

- T1: the onset timing of the active cable retraction.

- T2: the completion timing of the active cable retraction.

- T3: the start timing of the cable release.

- PosOffset: Bowden cable position right before the active cable retraction (at $\mathbf{T 1}$ ).

- PosMax: Bowden cable position when the cable is fully retracted (from $\mathbf{T} 2$ to $\boldsymbol{T} 3$ ).

\section{Multi-articular controller (MA)}

The controller for the multi-articular load path was designed to deliver the majority of assistance during ankle push-off [34]. As shown in Fig. 2, the controller first detected the heel strike using the first peak of sagittal-plane gyro signal from the foot IMU, which happens approximately at $5 \%$ GC [34]. This event was used as the start of the multi-articular controller, $\boldsymbol{T} \boldsymbol{O}_{M A}$ (i.e. $\boldsymbol{T} \mathbf{O}_{M A}=5 \% \mathrm{GC}$ ), and starting from $\mathbf{T} \boldsymbol{0}_{M A}$, the motor shortened the cable up to PosOffset $_{M A}$ at a constant speed of $394 \mathrm{~mm} \mathrm{~s}^{-1}$ (65\% of the maximum cable speed of the multi-articular actuator unit). Note that this cable speed was determined during pilot experiments to strike balance between being fast enough to reach PosOffset $_{M A}$ during early stance and not being excessively fast to not restrict the wearer's dorsiflexion. After reaching PosOffset $_{M A}$, the controller maintained this cable position until the onset of the active cable retraction, $\boldsymbol{T} \mathbf{1}_{M A}$ (automatically tuned; details in the following section). Then, the cable was actively retracted up to Pos$\operatorname{Max}_{M A}$ until the completion timing of the active cable retraction, $T 2_{M A}$ (automatically tuned; details in the following section). Then, the controller held the cable position constant until the load cell detects a force drop as the ankle further plantarflexes, and this event was used as the start of the cable release, $T 3_{M A}$. Starting from $T 3_{M A}$, the motor released the cable at the maximum speed of $606 \mathrm{~mm} \mathrm{~s}^{-1}$ to its zero position where the cable is completely slack, in order to not restrict the wearer during swing phase. After reaching the zero position, the motor maintained this cable position until the next heel strike detection.

At the end of each stride, the controller either increased or decreased PosOffset MA $_{\text {and }}$ asMax PaA $_{M A}$ for the next stride by comparing the desired and the measured force. For example, PosOffset $_{M A}$ was adjusted to deliver a peak force of $75 \mathrm{~N}$ (equivalent joint moment of approximately $7.5 \mathrm{Nm}$ ) between $\boldsymbol{T} \boldsymbol{0}_{M A}$ and $\boldsymbol{T} \mathbf{1}_{\boldsymbol{M A}}$, to consistently pretension the cable before the active cable retraction. Pos$\operatorname{Max}_{M A}$ was adjusted to deliver a peak force of $400 \mathrm{~N}$ (equivalent joint moment of approximately $40 \mathrm{Nm}$ ) between $T 1_{M A}$ and $T 3_{M A}$, as a primary means to deliver assistance during the active cable retraction.

\section{Hip extension controller (HE)}

The controller for the hip extension load path aimed at applying assistance in early stance while hip extensor muscles are active. The hip extension controller used constant timing parameters $\left(T 0_{H E}, T 1_{H E}, T 2_{H E}, T 3_{H E}\right)$ for all users without parameter tuning, whose values were from the experimental condition with the largest metabolic benefit in Ding et al. where the effect of four different sets of timing parameters were compared for an exosuit assisting hip extension [35]. Unlike the multi-articular controller, these timing parameters are represented as percentage of a gait cycle segmented by maximum hip flexion event (\% $\mathrm{GC}_{\mathrm{MHF}}$ ) detected by the thigh IMUs; note that the maximum hip flexion happens approximately $12 \%$ earlier than heel strike (i.e. $\% \mathrm{GC}_{\mathrm{MHF}} \approx \% \mathrm{GC}-12 \%$ ) [35]. As shown in Fig. 3, the controller first detected the maximum hip flexion using the thigh IMU and used this event as the start of the controller (i.e. $\mathbf{T} \boldsymbol{0}_{\boldsymbol{H E}}=0 \%$ $\left.\mathrm{GC}_{\mathrm{MHF}}\right)$. Then, the motor was controlled to shorten the cable up to PosOffset $_{H E}$ at a constant speed of $800 \mathrm{~mm} \mathrm{~s}^{-1}$, which was the maximum cable speed of the hip extension actuator. After reaching PosOff$\boldsymbol{s e t}_{\boldsymbol{H E}}$, the controller maintained this cable position until $7 \% \mathrm{GC}_{\mathrm{MHF}}$ (i.e. $\boldsymbol{T 1}_{\boldsymbol{H E}}=7 \% \mathrm{GC}_{\mathrm{MHF}}$ ). Then, the motor further retracted the cable up to $\boldsymbol{P o s M a x}_{\boldsymbol{H E}}$ until $28 \% \mathrm{GC}_{\mathrm{MHF}}$ (i.e. $\boldsymbol{T} 2_{H E}=28 \% \mathrm{GC}_{\mathrm{MHF}}$ ), and held the cable in this position until $34 \% \mathrm{GC}_{\mathrm{MHF}}$ (i.e. $\mathbf{T} \boldsymbol{3}_{\boldsymbol{H E}}$ $=34 \%\left(\mathrm{GC}_{\mathrm{MHF}}\right)$. Finally, the motor released the cable 
to its zero position, using the maximum cable speed of $800 \mathrm{~mm} \mathrm{~s}^{-1}$ similarly to the multi-articular controller. The PosOffset PE $_{H E}$ and PosMax ${ }_{H E}$ were adjusted at the end of each gait cycle, to deliver a peak force of $10 \mathrm{~N}$ (equivalent joint moment of approximately $1 \mathrm{Nm}$ ) between $\boldsymbol{T} \boldsymbol{0}_{\boldsymbol{H E}}$ and $\boldsymbol{T} \boldsymbol{1}_{\boldsymbol{H}}$ and a peak force of $300 \mathrm{~N}$ (equivalent joint moment of approximately $30 \mathrm{Nm}$ ) between $\boldsymbol{T} \mathbf{1}_{\boldsymbol{H E}}$ and $\boldsymbol{T} \mathbf{3}_{\boldsymbol{H E}}$, respectively.

\section{Augmentation-power-based control parameter tuning}

In order to customize the multi-articular assistance for each individual, an online parameter tuning method was developed which searches the control parameters that maximize the positive augmentation power delivered at the ankle. This assumes that the positive ankle augmentation power can be an indicator of the magnitude of assistance delivered at the ankle, which in turn may have a positive correlation with the corresponding metabolic benefit [6, 10, 19, 29-33]. Of note, in this study the average positive augmentation power was calculated by dividing the positive augmentation work over a gait cycle by the stride time. The positive augmentation work may also indicate the amount of assistance delivered to the joint, but it may significantly vary with the wearer's cadence. In contrast, positive power is less affected by variability in cadence, making it a more robust objective metric for control parameter tuning (See Additional file 1 for further discussion).

\section{Tuning parameter selection}

Among the control parameters defining the cable position profile of the multi-articular controller, $\boldsymbol{T} \mathbf{1}_{\boldsymbol{M A}}$ (onset timing of the active cable retraction) and $D_{M A}\left(T 2_{M A}-T 1_{M A}\right.$; duration of the active cable retraction) were selected as the parameters to be tuned for each individual. As highlighted in green in Fig. 2, these parameters play an important role in determining the cable position profile during the active cable retraction phase, where the majority of ankle assistance is delivered during push off. In addition, in pilot experiments $T 1_{M A}$ and $D_{M A}$ showed higher sensitivity to the changes in positive ankle augmentation power than other control parameters, highlighting the importance of customization of these parameters. The initial parameter ranges were set to $35-50 \%$ GC for $\boldsymbol{T} \boldsymbol{1}_{\boldsymbol{M A}}$ and $7.5-22.5 \%$ GC for $D_{M A}$, where the actuation system could generate the desired level of peak assistive force $(400 \mathrm{~N})$ at the ankle joint. With this parameter range the multi-articular controller was capable of creating force profiles ranged approximately from 35 to $65 \% \mathrm{GC}$, which sufficiently covers the phase of positive biological ankle power while walking.

\section{Positive augmentation power measurement}

While walking with the exosuit active, the instantaneous ankle augmentation power was calculated from the ankle joint velocity (measured by the foot and shank IMUs) and the assistive force (measured by the multi-articular load cell), assuming a constant lever arm of $10 \mathrm{~cm}$ at the ankle. The positive augmentation work over a stride was calculated by integrating the positive area under the instantaneous power curve over a gait cycle, and finally the positive augmentation power was calculated by dividing the positive work by the stride time $[6,9,30,34,36]$. In this study, while each parameter setting was given to the wearer for 45 strides, the positive augmentation power for each condition was averaged over the last 30 strides (Note that, in pilot experiments, it took about 10 strides to reach a steady-state positive augmentation power value when a new set of parameters were applied).

\section{Online parameter tuning algorithm}

A simple online parameter tuning algorithm based on 2-D grid search similar to gradient descent was developed and used for this study. During the tuning process, subjects continuously walked with the exosuit on a treadmill, and the multi-articular controller applied 16 different parameter settings in series, searching the parameter values that maximize the positive augmentation power delivered at the ankle. First, the controller swept the initial four conditions, where $\boldsymbol{T} \mathbf{1}_{\boldsymbol{M A}}$ was varied over $35,40,45$, and $50 \%$ GC while $\boldsymbol{D}_{\boldsymbol{M A}}$ was held constant at $15 \% \mathrm{GC}$. These values were chosen by varying $\boldsymbol{T} \mathbf{1}_{\boldsymbol{M A}}$ with $5 \%$ interval within its initial range (35$50 \% \mathrm{GC}$ ) while holding $\boldsymbol{D}_{M A}$ constant at the mid-point of its initial range $(7.5-22.5 \% \mathrm{GC})$. Among the four values of $T 1_{M A}$, the controller selected the setting where the largest positive augmentation power was delivered to the ankle. Of note, due to the hardware limitations (specifically motor power), during this selection step the controller was designed to exclude certain parameter settings where the exosuit was limited from achieving a desired peak force of $400 \mathrm{~N}$. Next, the controller applied another four conditions by varying $D_{M A}$ with $5 \%$ interval within its range (i.e. $\boldsymbol{D}_{M A}=7.5$, 12.5, 17.5, and 22.5\%) while holding $\boldsymbol{T} \mathbf{1}_{\boldsymbol{M A}}$ constant at the previously selected value. Similarly, among the four values of $D_{M A}$, the controller selected the value with the largest positive ankle augmentation power. Following this alternate parameter search scheme, another set of exploration over both $\boldsymbol{T} \mathbf{1}_{\boldsymbol{M A}}$ and $\boldsymbol{D}_{\boldsymbol{M A}}$ was repeated with a reduced interval of $2.5 \%$. Finally, the parameter setting where the positive ankle augmentation power was maximized among the 16 conditions was chosen for each individual. Of note, the total number of conditions included in the tuning process was determined during pilot experiments, where we found that a modification of control parameters smaller than $2.5 \%$ GC did not induce a substantial change in the positive ankle augmentation power. In addition, the total 16 conditions allowed for the entire tuning process to be done in about $15 \mathrm{~min}$, which is 
short enough to not induce significant fatigue of the wearer during the continuous walking trial.

This relatively simple parameter tuning algorithm presents several positive attributes. At this stage, the focus of the study was on testing the feasibility of the augmentation-power-based control parameter tuning approach, so a simple method aimed at proving the general concept was preferred, as opposed to applying a more sophisticated and efficient optimization technique. In addition, as this method sequentially varies either one of the two control parameters while holding the other constant, the tuning process is comprised of a series of single parameter sweeps. Compared to other multi-dimensional optimization techniques that may vary multiple parameters at the same time, this approach yields data that may provide insight on the individual parameter's effect during the tuning process.

\section{Experimental protocol}

Seven healthy male adults with prior experience walking with the exosuit participated in this study (age 31.0 \pm 7.3 years; mass $83.0 \pm 7.9 \mathrm{~kg}$; height $1.80 \pm 0.04 \mathrm{~m}$; mean \pm SD). The Harvard Longwood Medical Area Institutional Review Board approved the study, and all participants provided written informed consent. The study consisted of a single-day experimental session, involving walking on a treadmill at $1.50 \mathrm{~m} \mathrm{~s}^{-1}$ carrying a loaded military rucksack with a total mass of $6.8 \mathrm{~kg}$, with and without wearing the exosuit (Fig. 4). Note that a fixed walking speed was used in this study, because variation in walking speed by itself may change the positive ankle augmentation power. The amount of load carried during this experiment was selected to not induce fatigue in the participants. At the beginning of the session, a 4-min standing trial was performed to collect steady-state standing metabolic cost. Participants then underwent a 15-min control parameter

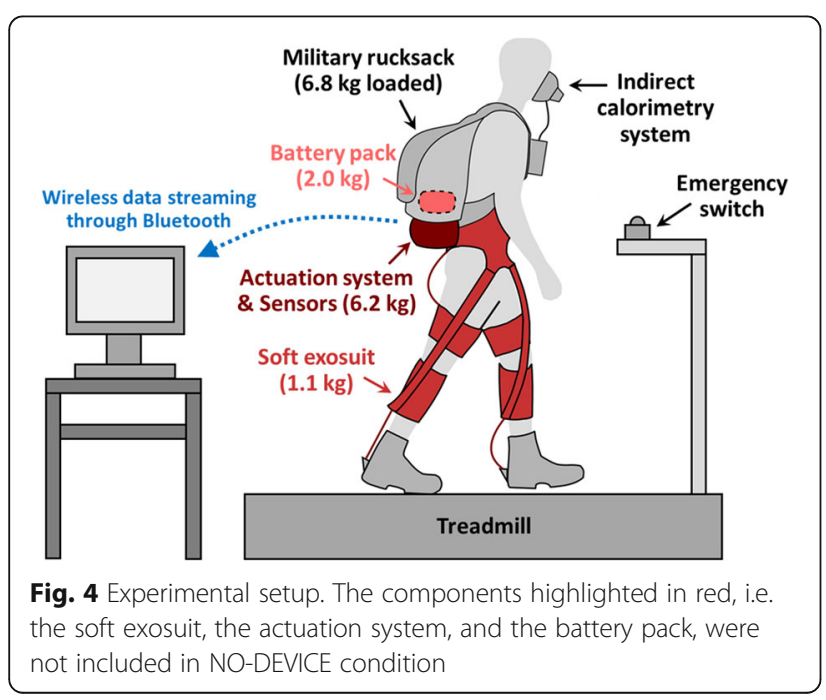

tuning process explained above. After the tuning process, participants completed three 5-min experimental conditions: loaded walking with the exosuit unpowered (EXO-OFF), loaded walking with the exosuit active using individualized parameters found by the augmentation-power-based parameter tuning (EXO-ON), and loaded walking without wearing the exosuit (NO-DEVICE). The two exosuit conditions were randomized, but NO-DEVICE condition was always completed last to minimize exosuit donning and doffing time during the session.

\section{Measurement and data processing}

A portable indirect calorimetry system (K4b2, COSMED, Rome, Italy) was used to measure the metabolic cost of walking. Metabolic power was calculated using a modified Brockway equation [37] and averaged over the last $2 \mathrm{~min}$ of each condition. For each walking condition, net metabolic rate was calculated by subtracting the metabolic power during standing and then normalized to each participant's body mass. Percent net metabolic benefit was calculated as the reduction in net metabolic rate for EXO-ON condition compared to NO-DEVICE condition, while gross metabolic benefit was calculated compared to EXO-OFF condition:

$$
\begin{aligned}
& \text { Net Metabolic Benefit }[\%] \\
& \qquad=\frac{\left(N O_{D E V I C E}\right)-\left(E_{X X} O_{O N}\right)}{\left(N O_{D E V I C E}\right)-(\text { STANDING })} \times 100 \%
\end{aligned}
$$

$$
\begin{aligned}
& \text { Gross Metabolic Benefit }[\%] \\
& =\frac{(\text { EXO_OFF })-(\text { EXO_ON })}{(\text { EXO_OFF })-(\text { STANDING })} \times 100 \%
\end{aligned}
$$

Inter-subject mean and standard error of the mean (SEM) were calculated for the net metabolic rate and the percent metabolic reduction. Two-sided paired t-tests (significance level $\alpha=0.01$; MATLAB, MathWorks Inc., Natick, MA, USA) were used to test statistical significance of the difference in net metabolic rate between two conditions.

\section{Results}

\section{Positive ankle augmentation power map}

Figure 5 shows a positive ankle augmentation power map of a representative subject (S5) from the parameter tuning process. As shown, the controller explored a wide range of conditions and found the parameter set $\left(\boldsymbol{T} \mathbf{1}_{\boldsymbol{M A}}=43.75 \%, \boldsymbol{D}_{\boldsymbol{M A}}=17.5 \%\right)$ that delivered the most positive augmentation power at the ankle (unilateral $8.70 \mathrm{~W}$ ), which was $83 \%$ higher than the lowest condition (unilateral $4.76 \mathrm{~W}$ at $\boldsymbol{T} \mathbf{1}_{\boldsymbol{M A}}=35 \%, \boldsymbol{D}_{\boldsymbol{M A}}=15 \%$ ). 


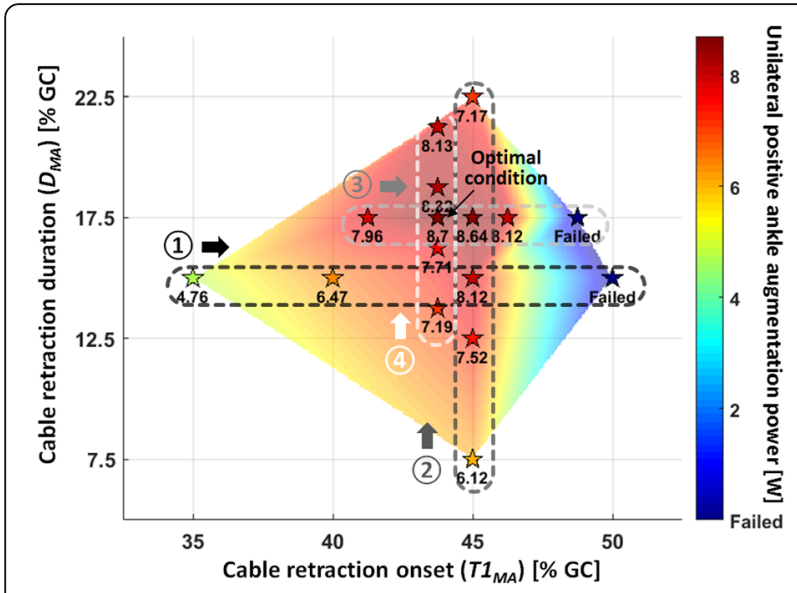

Fig. 5 Positive ankle augmentation power map of a representative subject (S5) from the control parameter tuning process. Stars indicate the conditions that the controller explored, and the number below each star indicates the unilateral positive augmentation power delivered at the ankle for each condition in watts. The arrows with numbers in circles (1), (2), (3), and (4)) indicate the sequence of the exploration, along the grouped conditions indicated by the dotted lines. The stars labelled as "Failed" indicate the conditions that were excluded as the exosuit was limited from achieving a desired peak force of $400 \mathrm{~N}$

\section{Subject-specific control parameters}

Figure 6 shows the subject-specific control parameters found by the parameter tuning method and the resultant force profiles. As shown in Fig. 6a, the wearer-specific cable retraction onset $\left(\boldsymbol{T} 1_{M A}\right)$ ranged from 43.75 to $46.25 \%$

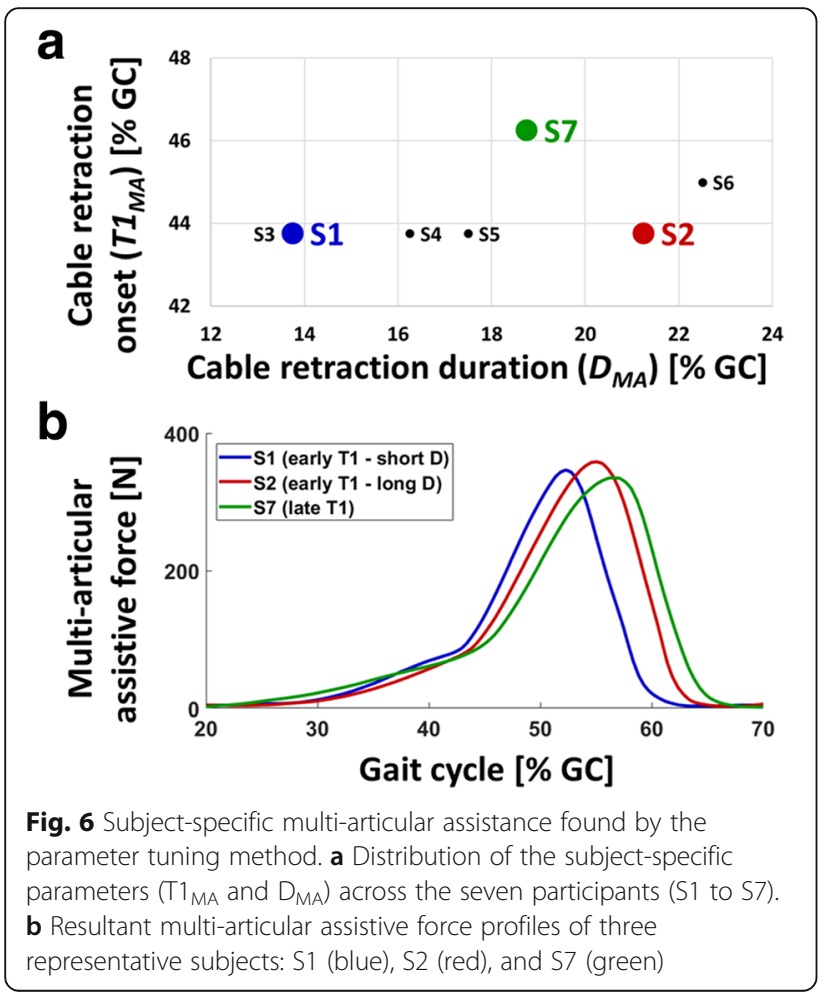

of a gait cycle, whereas the cable retraction duration $\left(\boldsymbol{D}_{M A}\right)$ ranged from 13.75 to $22.5 \%$ of a gait cycle across the participants. These differences in cable retraction timing resulted in a wide range of subject-specific force profiles for multi-articular assistance, as shown in Fig. 6b.

\section{Metabolic cost}

Walking with the autonomous multi-joint soft exosuit with individualized parameters significantly improved the energy economy of load carriage for all participants (Table 1). As shown in Fig. 7, the net metabolic rate of loaded walking in NO-DEVICE, EXO-OFF, and EXO-ON conditions were $4.35 \pm 0.24 \mathrm{~W} / \mathrm{kg}, 4.74 \pm$ $0.18 \mathrm{~W} / \mathrm{kg}$, and $3.70 \pm 0.21 \mathrm{~W} / \mathrm{kg}$ (mean $\pm \mathrm{SEM})$, respectively. This corresponds to a significant net metabolic reduction of $14.88 \pm 1.09 \%$ (paired t-test, $P=5 \times 10^{-5}$ ) and a significant gross metabolic reduction of $22.03 \pm$ $2.23 \%\left(P=2 \times 10^{-5}\right)$.

\section{Conclusion}

In this paper, we present an autonomous multi-joint soft exosuit and an online parameter tuning approach that customizes assistance for each individual based on the positive power delivered at the ankle. The parameter tuning method was capable of automatically finding the wearer-specific control parameters using exosuit sensors, demonstrating its potential to customize an assistive device outside of the lab so as to maximize positive augmentation power at the ankle. The subject-specific control parameters resulted in a wide range of assistance profiles, which supports the growing recognition of the importance of such control individualization for assistive devices. Additionally, the autonomous multi-joint exosuit with the subject-specific control parameters significantly reduced the net metabolic cost of loaded walking by $14.88 \%$, relative to walking without wearing the device.

Despite a significant metabolic benefit, there are a number of limitations of this study worth mentioning.

Table 1 Metabolic result for each participant

\begin{tabular}{lllllll}
\hline Subject & $\begin{array}{l}\text { STANDING } \\
{[\mathrm{W} / \mathrm{kg}]}\end{array}$ & $\begin{array}{l}\text { NO- } \\
\text { DEVICE } \\
{[\mathrm{W} / \mathrm{kg}]}\end{array}$ & $\begin{array}{l}\text { EXO- } \\
\text { OFF } \\
{[\mathrm{W} / \mathrm{kg}]}\end{array}$ & $\begin{array}{l}\text { EXO-ON } \\
{[\mathrm{W} / \mathrm{kg}]}\end{array}$ & $\begin{array}{l}\text { Net } \\
\text { metabolic } \\
\text { benefit [\%] }\end{array}$ & $\begin{array}{l}\text { Gross } \\
\text { metabolic } \\
\text { benefit [\%] }\end{array}$ \\
\hline S1 & 1.267 & 5.039 & 5.643 & 4.423 & $16.34 \%$ & $27.88 \%$ \\
S2 & 1.025 & 4.975 & 5.172 & 4.457 & $13.12 \%$ & $17.26 \%$ \\
S3 & 1.331 & 6.306 & 6.537 & 5.334 & $19.55 \%$ & $23.11 \%$ \\
S4 & 1.707 & 6.258 & 6.613 & 5.705 & $12.15 \%$ & $18.50 \%$ \\
S5 & 1.319 & 6.288 & 6.681 & 5.728 & $11.27 \%$ & $17.77 \%$ \\
S6 & 1.486 & 4.929 & 5.768 & 4.394 & $15.53 \%$ & $32.08 \%$ \\
S7 & 1.572 & 6.362 & 6.444 & 5.586 & $16.18 \%$ & $17.60 \%$ \\
$\begin{array}{l}\text { Mean } \\
( \pm \text { SEM) }\end{array}$ & & & & & $14.88 \%$ & $22.03 \%$ \\
\hline
\end{tabular}




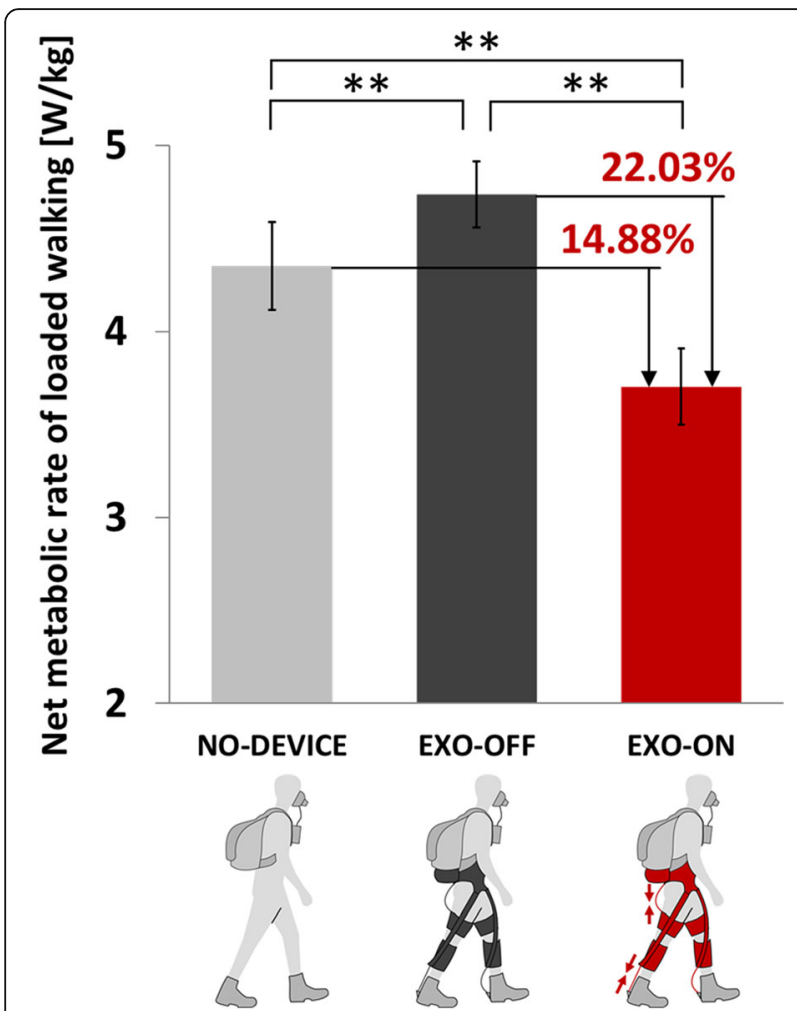

Fig. 7 Metabolic cost of load carriage for the three experimental conditions. Solid bars indicate inter-subject mean of net metabolic rate of loaded walking, while error bars indicate SEM. Double asterisks (**) indicate that the difference between the two conditions is statistically significant (paired t-test; $n=7 ; P<0.01$ ). For EXO-ON, the net metabolic rate of loaded walking was significantly reduced by $14.88 \pm 1.09 \%$ compared to NO-DEVICE $(P=5 \times 10-5)$ and by $22.03 \pm 2.23 \%$ compared to EXO-OFF $(P=2 \times 10-5)$

First, although the capability of the control parameter tuning method to maximize the positive ankle augmentation power was demonstrated, the amount of metabolic improvement produced by using the subject-specific parameters was not clear. At this stage, the focus was on proving the general feasibility of the approach, and the experiment that we conducted was not able to isolate the effect of the control parameter tuning. Follow-up studies will investigate the metabolic landscape versus control parameters, bridging the gap between the augmentation-power-based parameter tuning at the ankle and the whole-body energetics. Second, the parameter tuning algorithm was based on a simple grid search similar to gradient descent, which may be vulnerable to existence of local minima or measurement noise. Future research will explore the use of statistical optimization algorithms which can search the global optimum in a large parameter space, such as Bayesian optimization [20] or simulated annealing [38], to make the tuning process more reliable and robust. In addition, the joint and muscle-tendon level mechanisms that contributed to this high metabolic reduction is not yet clear due to limited biomechanical and physiological measurements. In follow-up studies, we will include more comprehensive measurements, such as 3-D motion capture, electromyography, and ultrasound imaging, to further investigate how the exosuit and the wearer interact with each other. Lastly, whereas only a specific walking condition was tested in this study, future studies will evaluate the efficacy of the device in various conditions, such as walking at different walking speeds with different loads. This may provide insights on developing a parameter tuning method suitable for overground walking where the wearer may continuously change their walking speed, enabling potential use of this parameter tuning method for patient populations $[39,40]$.

\section{Additional file}

Additional file 1: Variability of positive augmentation power and work with cadence. (PDF $285 \mathrm{~kb}$ )

\section{Abbreviations \\ \% GC: Percentage of a gait cycle (segmented by heel strike); \% \\ $\mathrm{GC}_{\mathrm{MHF}}$ : Percentage of a gait cycle segmented by maximum hip flexion; HE (subscription): Parameters for hip extension controller; IMU: Inertial measurement unit; MA (subscription): Parameters for multi-articular controller;} SD: Standard deviation; SEM: Standard error of the mean

\section{Acknowledgments}

The authors would like to thank Danielle Louise Ryan, Taylor Greenberg Goldy, David Perry, Asa Eckert-Erdheim, Patrick Murphy, Giuk Lee, Dabin Choe, Maria Athanassiu, Brice Mikala Iwangou, Fausto A. Panizzolo, and Sarah Sullivan for their contribution to this work.

\section{Funding}

This material is based upon the work supported by the Defense Advanced Research Projects Agency (DARPA), Warrior Web Program (W911NF-14-C-0051). This work was also partially funded by the National Science Foundation (CNS-1446464), the Wyss Institute for Biologically Inspired Engineering, and the John A. Paulson School of Engineering and Applied Sciences at Harvard University. S.L. and J.K. are recipients of the Samsung Scholarship.

\section{Availability of data and materials \\ All data generated or analyzed during this study are included in this published article.}

\section{Authors' contributions}

SL, JK, NK, NM, IG, and CJW developed the controller. SL, JK, AL, IG, and CJW developed the control parameter tuning method. SL, JK, LB, AL, IG, and CJW designed the experiment. $S L$ and $L B$ performed the experiment. $S L$, $L B$, and CJW analyzed and interpreted the data. SL, JK, LB, and CJW prepared the manuscript. All authors provided critical feedback on the manuscript. All authors read and approved the final manuscript.

Ethics approval and consent to participate

The study was approved by the Harvard Longwood Medical Area Institutional Review Board, and all methods were carried out in accordance with the approved study protocol. All participants provided written informed consent before their participation, and after the nature and possible consequences of the study were explained.

Consent for publication

Not applicable. 


\section{Competing interests}

Patents have been filed with the U.S. Patent Office by Harvard University, which describe the exosuit components documented in this manuscript. SL, JK, NK, NM, IG, and CJW are authors of those patents. Harvard University has entered into a license and collaboration agreement with ReWalk Robotics. CJW is a paid consultant for ReWalk Robotics.

\section{Publisher's Note}

Springer Nature remains neutral with regard to jurisdictional claims in published maps and institutional affiliations.

\section{Received: 15 December 2017 Accepted: 3 July 2018}

\section{Published online: 13 July 2018}

\section{References}

1. Ferris DP, Gordon KE, Sawicki GS, Peethambaran A. An improved powered ankle-foot orthosis using proportional myoelectric control. Gait Posture. 2006;23:425-8.

2. Walsh CJ, Endo K, Herr H. A quasi-passive leg exoskeleton for load-carrying augmentation. Int J Human Robot. 2007;04:487-506.

3. Sup F, Bohara A, Goldfarb M. Design and control of a powered Transfemoral prosthesis. Int J Robot Res. 2008;27:263-73.

4. Sawicki GS, Ferris DP. A pneumatically powered knee-ankle-foot orthosis (KAFO) with myoelectric activation and inhibition. J Neuroeng Rehabil. 2009;6:23

5. van Dijk W, van der Kooij H, Hekman E. A passive exoskeleton with artificial tendons: design and experimental evaluation. In: IEEE International Conference on Rehabilitation Robotics (ICORR); 2011.

6. Mooney LM, Rouse EJ, Herr HM. Autonomous exoskeleton reduces metabolic cost of human walking during load carriage. J Neuroeng Rehabil. 2014;11:80.

7. Malcolm P, Derave W, Galle S, De Clercq D. A simple exoskeleton that assists plantarflexion can reduce the metabolic cost of human walking. PLoS One. 2013;8:e56137.

8. Collins SH, Wiggin MB, Sawicki GS. Reducing the energy cost of human walking using an unpowered exoskeleton. Nature. 2015;522:212-5.

9. Koller JR, Jacobs DA, Ferris DP, Remy CD. Learning to walk with an adaptive gain proportional myoelectric controller for a robotic ankle exoskeleton. J Neuroeng Rehabil. 2015;12:97.

10. Mooney LM, Herr HM. Biomechanical walking mechanisms underlying the metabolic reduction caused by an autonomous exoskeleton. J Neuroeng Rehabil. 2016;13:4.

11. Zhang J, Fiers P, Witte KA, Jackson RW, Poggensee KL, Atkeson CG, Collins SH. Human-in-the-loop optimization of exoskeleton assistance during walking. Science. 2017;23:1280-4.

12. Lee J, Seo K, Lim B, Jang J, Kim K, Choi H. Effects of assistance timing on metabolic cost, assistance power, and gait parameters for a hip-type exoskeleton. In: IEEE International Conference on Rehabilitation Robotics (ICORR); 2017.

13. Asbeck AT, De Rossi SMM, Holt KG, Walsh CJ. A biologically inspired soft exosuit for walking assistance. Int J Robot Res. 2015;34:744-62.

14. Asbeck AT, Schmidt K, Galiana I, Wagner D, Walsh CJ. Multi-joint soft exosuit for gait assistance. In: IEEE International Conference on Robotics and Automation (ICRA); 2015

15. Panizzolo FA, Galiana I, Asbeck AT, Siviy C, Schmidt K, Holt KG, Walsh CJ. Biologically-inspired multi-joint soft exosuit that can reduce the energy cost of loaded walking. J Neuroeng Rehabil. 2016;13:43.

16. Ding Y, Galiana I, Asbeck AT, De Rossi SMM, Bae J, Santos TRT, Araujo VL, Lee S, Holt KG, Walsh C. Biomechanical and physiological evaluation of multi-joint assistance with soft exosuits. Trans Neural Syst Rehabil Eng. 2017; 25:119-30.

17. Quinlivan BT, Lee S, Malcolm P, Rossi DM, Grimmer M, Siviy C, Karavas N, Wagner D, Asbeck A, Galiana I, Walsh CJ. Assistance magnitude versus metabolic cost reductions for a tethered multiarticular soft exosuit. Sci Robot. 2017;2(2):eaah4416.

18. Lee G, Kim J, Panizzolo FA, Zhou YM, Baker LM, Galiana I, Malcolm P, Walsh CJ. Reducing the metabolic cost of running with a tethered soft exosuit. Sci Robot. 2017:2:eaan6708.

19. Malcolm P, Lee S, Crea S, Siviy C, Saucedo F, Galiana I, Panizzolo FA, Holt KG, Walsh CJ. Varying negative work assistance at the ankle with a soft exosuit during loaded walking. J Neuroeng Rehabil. 2017;14:62.
20. Ding Y, Kim M, Kuindersma S, Walsh CJ. Human-in-the-loop optimization of hip assistance with a soft exosuit during walking. Sci Robot. 2018;3:eaar5438.

21. Felt W, Selinger JC, Donelan JM, Remy CD. 'Body-in-the-loop': optimizing device parameters using measures of instantaneous energetic cost. PLoS One. 2015;10:e0135342.

22. Kim M, Ding Y, Malcolm P, Speeckaert J, Siviy CJ, Walsh CJ, Kuindersma S. Human-in-the-loop Bayesian optimization of wearable device parameters. PLoS One. 2017;12:e0184054.

23. Aghasadeghi N, Zhao H, Hargrove LJ, Ames AD, Perreault EJ, Bretl T. Learning impedance controller parameters for lower-limb prostheses. In: International Conference on Intelligent Robots and Systems (IROS); 2013.

24. Spanias JA, Simon AM, Hargrove LJ. Across-user adaptation for a powered lower limb prosthesis. In: International Conference on Rehabilitation Robotics (ICORR); 2017.

25. Kumar S, Mohammadi A, Gans N, Gregg RD. Automatic tuning of virtual constraint-based control algorithms for powered knee-ankle prostheses. In: IEEE Conference on Control Technology and Applications (CCTA); 2017.

26. Wen Y, Si J, Gao X, Huang S, Huang H. A new powered lower limb prosthesis control framework based on adaptive dynamic programming. IEEE Trans Neural Netw. 2017;28:2215-20.

27. Huang $\mathrm{H}$, Crouch DL, Liu M, Sawicki GS, Wang D. A cyber expert system for auto-tuning powered prosthesis impedance control parameters. Ann Biomed Eng. 2016:44:1613-24.

28. Lee S, Karavas N, Quinlivan BT, Ryan DL, Perry D, Eckert-Erdheim A, Murphy P, Greenberg Goldy T, Menard N, Athanassiu M, Kim J, Lee G, Galiana I, Walsh CJ. Autonomous multi-joint soft exosuit for assistance with walking Overground. In: IEEE international conference on robotics and automation (ICRA); 2018.

29. Jackson RW, Collins SH. An experimental comparison of the relative benefits of work and torque assistance in ankle exoskeletons. J Appl Physiol. 2015; 119:541-57.

30. Galle S, Malcolm P, Collins SH, De Clerca D. Reducing the metabolic cost of walking with an ankle exoskeleton: interaction between actuation timing and power. J Neuroeng Rehabil. 2017:14:35.

31. Malcolm P, Rossi DM, Siviy C, Lee S, Quinlivan BT, Grimmer M, Walsh CJ. Continuous sweep versus discrete step protocols for studying effects of wearable robot assistance magnitude. J Neuroeng Rehabil. 2017;14:72.

32. Grimmer M, Quinlivan BT, Lee S, Malcolm P, Rossi DM, Siviy C, Walsh CJ. Comparison of the human-exosuit interaction using ankle moment and ankle positive power inspired walking assistance. J Biomech. in review

33. Quinlivan B, Walsh CJ. System mass vs. augmentation power tradeoffs in Exoskeletons \& Exosuit. In: Dynamic walking conference (DWC); 2018.

34. Lee S, Crea S, Malcolm P, Galiana I, Asbeck A, Walsh C. Controlling negative and positive power at the ankle with a soft exosuit. In: IEEE International Conference on Robotics and Automation (ICRA); 2016

35. Ding Y, Galiana I, Siviy C, Panizzolo FA, Walsh C. IMU-based iterative control for hip extension assistance with a soft exosuit. In: IEEE International Conference on Robotics and Automation (ICRA); 2016.

36. Yandell MB, Quinlivan BT, Popov D, Walsh C, Zelik KE. Physical Interface dynamics Alter how robotic exosuits augment human movement: implications for optimizing wearable assistive devices. J Neuroeng Rehabil. 2017;14:40.

37. Brockway JM. Derivation of formulae used to calculate energy expenditure in man. Hum Nutr Clin Nutr. 1987:41:463-71.

38. Kirkpatrick $S$, Gelatt CD, Vecchi MP. Optimization by simulated annealing. Science. 1983:13(220):671-60

39. Awad LN, Bae J, O'Donnell K, De Rossi SMM, Hendron K, Sloot LH, Kudzia P, Allen S, Holt KG, Ellis TD, Walsh CJ. A soft robotic exosuit improves walking in patients after stroke. Sci Transl Med. 2017;9:eaai9084.

40. Bae J, Siviy C, Rouleau M, Menard N, O'Donnell K, Galiana I, Athanassiu M, Ryan D, Bibeau C, Sloot L, Kudzia P, Ellis T, Awad L, Walsh CJ. A lightweight and efficient portable soft exosuit for paretic ankle assistance in walking after stroke. In: IEEE international conference on robotics and automation (ICRA); 2018. 